\title{
CONF-960850--1
}

\section{Detection of the Normal Zone with Cowound Sensors in Cable-in Conduit Conductors}

Nicolai N. Martovetsky and Michael R. Chaplin

This paper was prepared for submittal to 1996 Applied Superconductivity Conference Pittsburgh, PA August 25-30, 1996

July 30, 1996

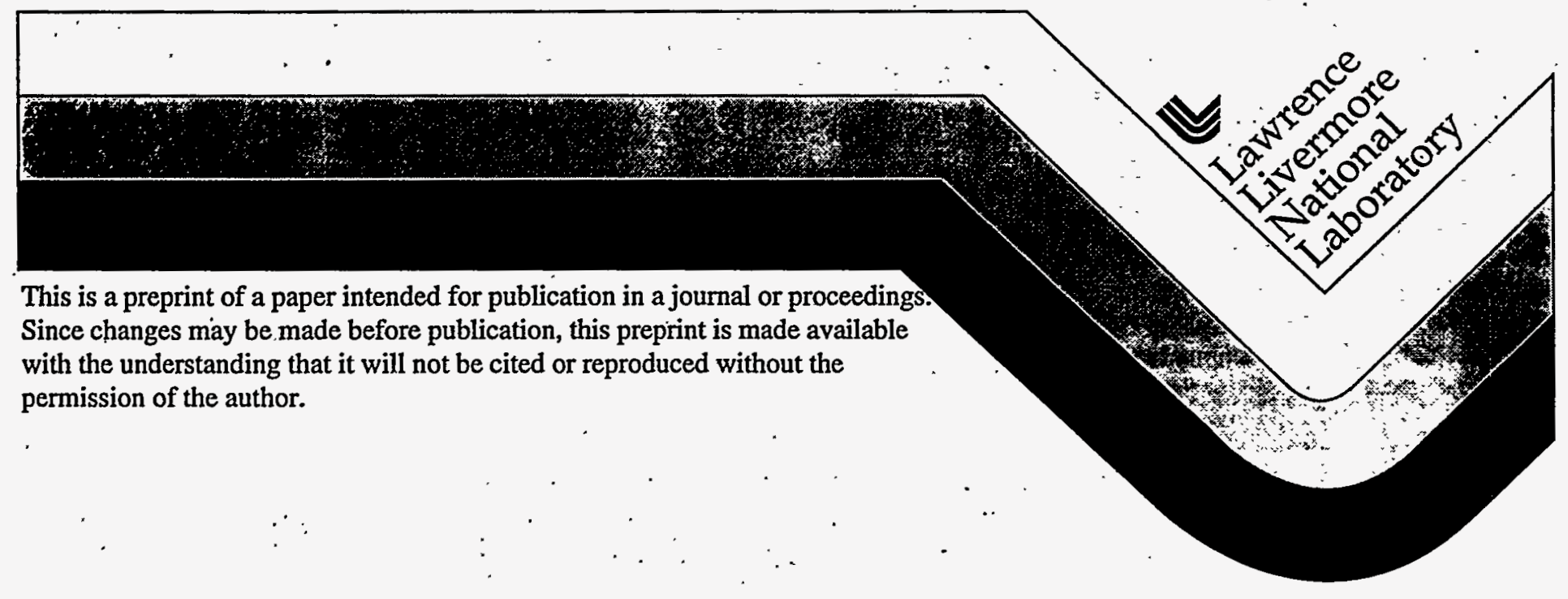

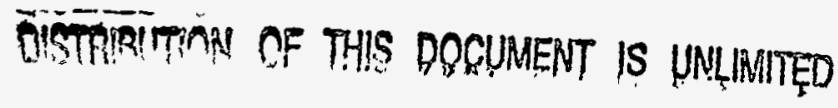




\section{DISCLAIMER}

This document was prepared as an account of work sponsored by an agency of the United States Government. Neither the United States Government nor the University of California nor any of their employees, makes any warranty, express or implied, or assumes any legal liability or responsibility for the accuracy, completeness, or usefulness of any information, apparatus, product, or process disclosed, or represents that its use would not infringe privately owned rights. Reference herein to any specific commercial product, process, or service by trade name, trademark, manufacturer, or otherwise, does not necessarily constitute or imply its endorsement, recommendation, or favoring by the United States Government or the University of California. The views and opinions of authors expressed herein do not necessarily state or reflect those of the United States Government or the University of California, and shall not be used for advertising or product endorsement purposes. 


\section{DISCLAIMER}

Portions of this document may be illegible in electronic image products. Images are produced from the best available original document. 


\title{
Detection of the Normal Zone with Cowound Sensors in Cable-in Conduit Conductors
}

\author{
Nicolai N. Martovetsky and Michael R. Chaplin \\ Lawrence Livermore National Laboratory, Livermore, CA, 94550
}

\begin{abstract}
Tokamaks in the future will use superconducting cable-in-conduit-conductors (CICC) in all Poloidal Field (PF) \& Toroidal Field (TF) magnets. Conventional quench detection, the measurement of small resistive normal-zone voltages $(<1 \mathrm{~V})$ in the magnets will be complicated by the presence of large inductive voltages $(>4$ $k V$ ). In the quench detection design for TPX, we have considered several different locations for internal co-wound voltage sensors in the cable cross-section as the primary mechanism to cancel this inductive noise. The Noise Rejection Experiment (NRE) at LLNL has been designed to evaluate which internal locations will produce the best inductive-noise cancellation, and provide us with experimental data for comparison with developed earlier theory. The details of the experiments and resulting data are presented and analyzed.
\end{abstract}

\section{INTRODUCTION}

The detection of a resistive normal-zone, or quench, in superconducting magnets has traditionally been accomplished by measuring the voltage across the resistance of the normal zone. Even in DC magnets though, the inductive voltages from di/dt can be several orders of magnitude greater than the resistive voltage component. To make this measurement possible, the inductive voltage must be reduced, or canceled, to a level less than the resistive component. Several techniques have been applied over the years to accomplish this; the most popular being the balanced-bridge scheme shown on the left in Fig. 1. During charging of the magnet, the resistor is adjusted to null the differential voitage, V1.

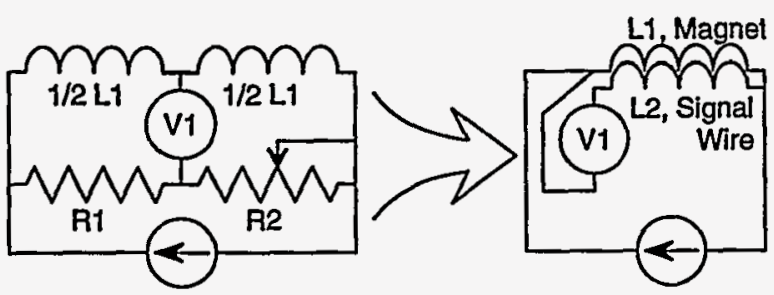

Fig. 1. Balanced-Bridge \& Co-Wound Sensor Quench Detection

When the primary inductive signals are the self di/dt of the magnet or of concentric sets of magnets, the balanced bridge

Manuscript received August 25, 1996.

This work was performed under the auspices of the U.S. Department of Energy by the Lawrence Livermore National Laboratory under contract No. W-7405-Eng-48 and the Princeton Plasma Physics Laboratory under contract number DE-AC02-76-CH03073. is effective at reducing the inductive-signal component by a factor of 1000 or more.In the magnet systems of a tokamak though, there are several sources of inductive noise that the balanced bridge would be ineffective at reducing sufficiently. Because of the complex field requirements of a tokamak, its magnets are exposed to time-varying, non-symmetrical transverse and parallel fields that a static balanced bridge can not compensate for. Ideally, what is needed is a second inductive element with the same dimensions and area of the magnet's winding pack, and the same twist pitches of the internal strands. The second inductor would then receive the exact same inductive signals from all sources that the magnet is exposed to. For the design of the Tokamak Physics Experiment (TPX) magnets, we investigated the use of cowound voltage sensors that would be used as shown on the right in Fig. 1.

\section{QUENCH-VOLTAGE DETECTION FOR TOKAMAKS}

\section{A. Magnet Parameters}

The tokamak magnets will endure large $\mathrm{dB} / \mathrm{dt}$ associated with the plasma initiation and any subsequent disruptions. Analysis $[3,4]$ indicates that $\mathrm{PF}$ s will experience a maximum of $12.8 \mathrm{~T} / \mathrm{s}$ and the TFs $22.3 \mathrm{~T} / \mathrm{s}$ from a fast plasma disruption in the TPX design. In a single null operating mode, the PF magnets will be exposed to as much as $4 \mathrm{kV}$ terminal-toterminal during the plasma initiation. Quench analyses [5] indicate that to discharge the magnets quickly enough to prevent the hot-spot temperature from rising above $150 \mathrm{~K}$, we must detect a normal zone voltage of $0.4-0.8 \mathrm{~V}$ in a maximum of one second. Reference [6] states that a 10:1 signal-to-noise ratio for the quench detection sensors must be attained to show feasibility of this sensing technique. With the numbers shown above, we see that the inductive noise signals (up to $4 \mathrm{kV}$ ) must be reduced to below $(0.4 \mathrm{~V} / 10=$ $0.04 \mathrm{~V}$ ) by the end the one-second detection window.

\section{B. Co-Wound Voltage Sensors}

An analysis [2] of several conductor locations for the cowound voltage sensors suggested the following locations to be considered for testing;

I. Twisted in the first stage of cabling (triplex) and therefore twisted with the same cabling pattern as all other strands, 
II. Placed outside the cable (but inside the conduit) in "valleys" of the last stage cabling and therefore twisted with the last stage cable,

III. Placed in the center of the last stage (3rd) subcable and therefore has the same twist pitch as the last cabling. stage,

IV. Placed in the geometric center of the CICC, and

V. Placed outside the sheath, but not twisted around the conductor.

TABLE I

TPX PF MAGNET CICC PARAMETERS

\begin{tabular}{|c|c|c|}
\hline Cable Stage & Cable Pattern & Twist Pitch \\
\hline 1 & 3 strands & $50 \mathrm{~mm}$ \\
\hline 2 & stage $1 \times 4$ & $100 \mathrm{~mm}$ \\
\hline 3 & stage $2 \times 5$ & $200 \mathrm{~mm}$ \\
\hline 4 & stage $3 \times 6$ & $400 \mathrm{~mm}$ \\
\hline
\end{tabular}

Sensor location $\mathrm{V}$ is the only option of applying a cowound sensor during magnet insulation; after the heattreatment phase for the conductor. However, it was ruled out early in our evaluation process because of the concern about electrical shorts from wires in the insulation pack. Below are results of analysis [2] and manufacturing considerations for sensors placement.

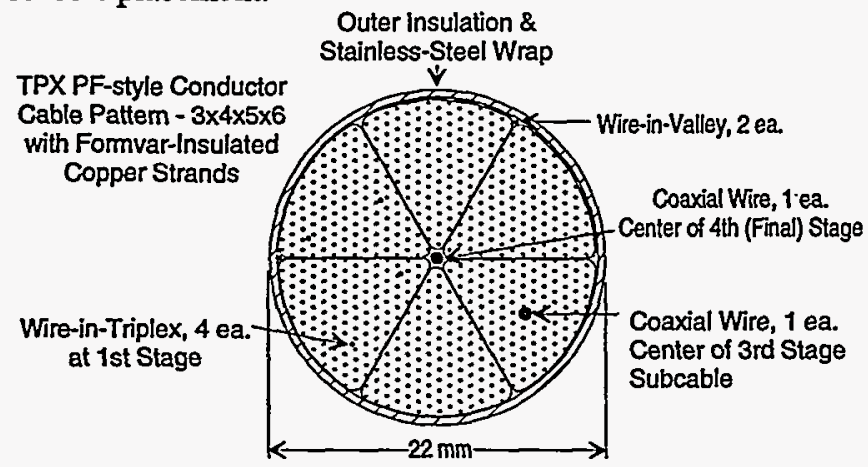

Fig. 2. Cable Configuration for the NRTC

Location IV is the easiest to manufacture or cable, but should be the least effective at picking up the same inductive voltages of the strands as it has none of the 4 stages of twist pitch associated with the strands.

Location III would be relatively simple to manufacture. With it having the same twist pitch as the last subcable, it should pickup most of the inductive voltages seen by the strands.

Location II is similar to IV as far as manufacturing; but may actually pickup more inductive voltage than the strands due to its loops having a larger area that the strands.

Location I would be difficult to manufacture if the other two strands in the triplex are $\mathrm{Nb}_{3} \mathrm{Sn}$. There is also speculation that this may cause problems for the superconducting strands of the triplex by not being able to transfer current to both of its triplex partners. However, location I should have the best match to the strands for inductive voltage pickup since its path through all cable stages ands twist pitches is the same. To eliminate possible conductor performance issues, one (or more) of the 1st-stage triplexes should be made up of three sensor wires.

\section{R\&D EXPERIMENTS}

\section{A. LLNL Noise-Rejection Experiment}

The LLNL Noise-Rejection Experiment (NRE) $[7,8]$ tested the noise-rejection capabilities of co-wound sensors (or wires) at each of the locations noted above. The $\mathrm{dB} / \mathrm{dt}$ testing conditions included external transverse, parallel, and self (di/dt) fields. Because the noise-coupling issues of signal wires located inside a cable are not dependent on temperature or resistivity, this experiment design with a room-temperature copper conductor should provide good test results for evaluating the effectiveness of the different signal-wire locations. The cable will use $3600.78 \mathrm{~mm}$ copper stands, and is shown in Fig. 2. with the location of the cowound voltage sensors.

The Noise-Rejection Test Coil (NRTC) is a layer wound solenoid with 36 turns. Conductor parameters were used to simulate the PF conductor parameters as shown in Table I. A Parallel-Field Coil (PFC) was wound as a toroid around the NRTC. This assembly was then placed in the bore of a pair of existing Transverse-Field Coils (TFCs) for testing, as shown in Fig. 3.

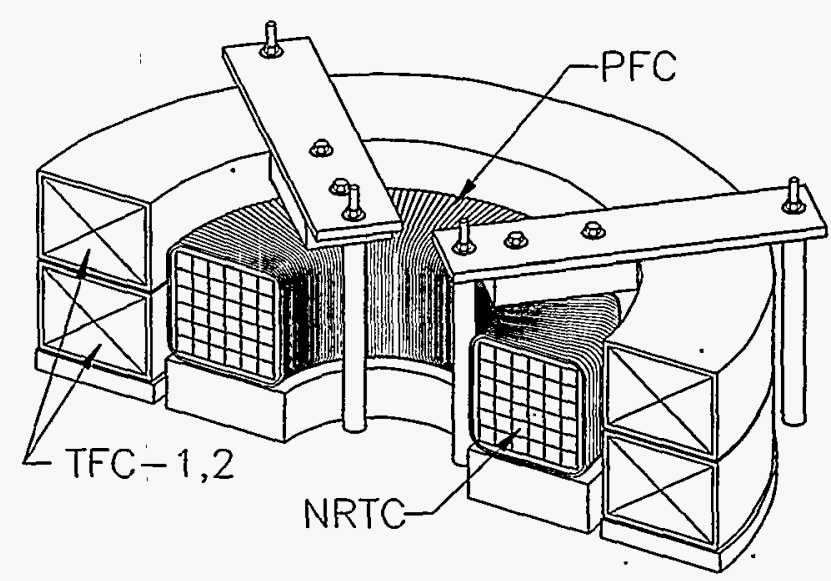

Fig. 3. Coil Configuration for the NRE

The current source for this experiment was the Japan Atomic Energy Research Institute (JAERD) Inductive-Heating Power Supply on loan to the FENIX Test Facility at LLNL. This power supply consists of a $2000 \mu \mathrm{F}$ capacitor bank with thyristor switches to "ring" the NRE coils individually during 
the experiment. The capacitor bank can be charged up to $1500 \mathrm{~V}$, and the thyristors can deliver current pulses up to $1000 \mathrm{~A}$.

-All voltage sensors are terminated with the copper strands into a clamped, copper block at terminal A of the NRTC. At the other end the copper strands are terminated into a similar copper block (terminal B), but with the voltage sensor wires extracted just before the block. These sensor wires are then paired with wires from the adjacent copper block and routed to the data acquisition system. In addition to these co-wound sensors, 2 pairs of conventional voltages taps are paired from the copper blocks to measure the full voltage that will appear across the NRTC as shown in Fig.4.

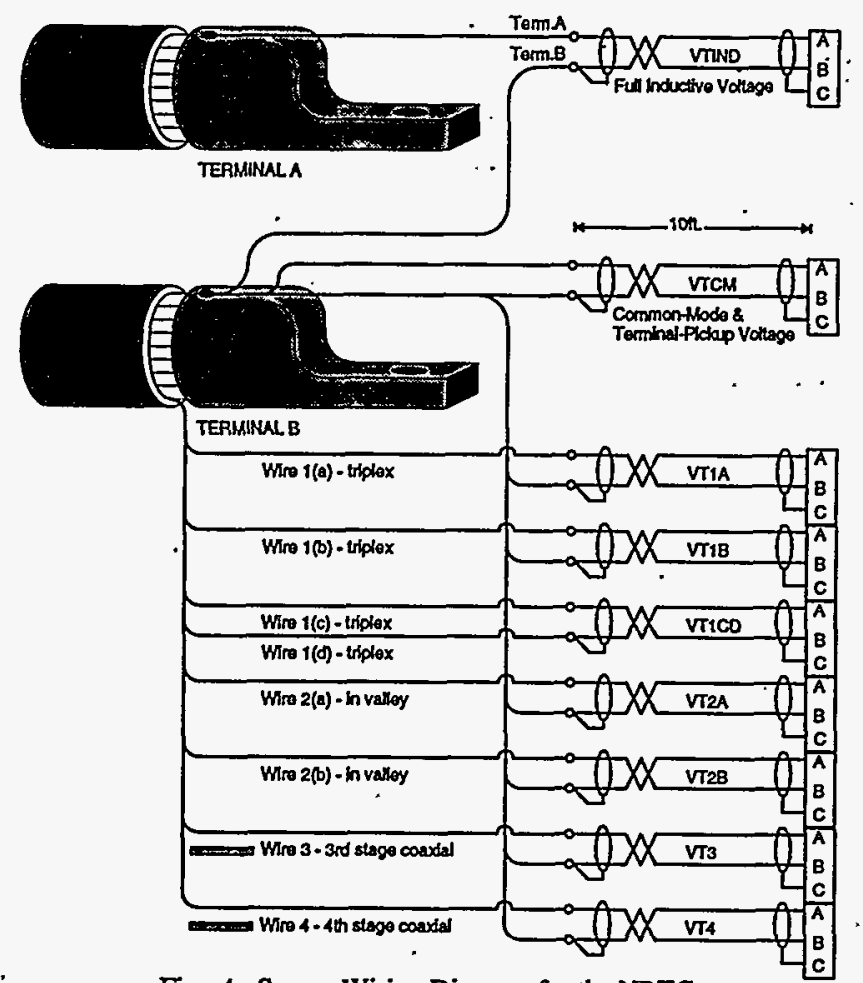

Fig. 4. Sensor Wiring Diagram for the NRTC

\section{B. NRE Results \& Analysis}

The resulting test conditions and data summary for the NRE are listed in Table II. The voltage taps (VTn) are numbered to indicate their location in the NRTC cable as stated above. $V_{\text {NRTC }}$ is the induced voltage across the NRTC as measured at its terminals. Rejection Factors are calculated as (VNTC/VTn). In the case of the parallel field, a rejection factor does not have the same meaning as for the other cases, even though we gave this parameter in the Table II.'The real figure of merit in the parallel field is how small this signal with the threshold value of the normal zone detection. As one can see from the Table II, only triplet sensor (location I) provides the signal negligible in comparison with the resistance of the normal zone on any reasonable length. For the self-field test mode, the IR voltage drop in the NRTC cable had to be subtracted from the raw signals to show the resultant inductive voltage term only.

As can be seen in the data below, the sensor in location IV (VT4) performs the worst with the sensor in location I (VT1) performing the best. Generally, VT3 was better than VT2; except in the parallel-field test where the larger VT2 radius makes a slight difference. Typical raw data from the 2-coil transverse test mode are shown in Figures. 5-6. In analyzing the NRE data, it was discovered that the multiple sensors at some locations were producing largely varying data. During a post experiment checkout, it was discovered that some sensors were shorted to some of the cable strands by multiple shorts via the stainless-steel tape. Evidently, the inner edge on the tape was turning down and after the NRTC was wound and assembled, the tape cut through the formvar insulation. Most of the discrepancy with the theory happened at the self field testing mode, since it is the only mode where we had to subtract resistive component from the total signal to evaluate inductive noise. As the location and resistance of the shorts was unknown, we calibrated these resistances at the DC mode to obtain the relationship between the resistive component of the voltage on each particular sensor with the short and the transport current. Knowing the resistances we could obtain the pure inductive signal by subtracting the resistive signal from the total signal from the sensor.

Table II shows the best results obtained with the different sensors. Variations in the signals from wires in different locations in some cases was quite large, especially for external pulsed field cases. This is understandable, as the reason for this signal is imperfection of the cabling pattern which is difficult to predict, especially for the conductors which cross section was reduced from round to the rectangular or square cross section. This is not fully unexpected and the data from NRE could help quantifying an extent of the cable imperfection in the terms of the coupling. of the individual strands with the magnetic flux from different sources (external transverse and parallel, self field) relative to the cable in average.

For example, the signal wire in the center of the cable showed the signal corresponding to its $0.5 \mathrm{~mm}$ displacement from the center. Sensor in the valley showed that it didn't stay exactly at the OD of the cable, but sunk inside by approximately $0.8 \mathrm{~mm}$. Effective radius of the sensor in the center of the last stage subcable (location III) was Rout $/ \sqrt{2}$, which corresponds to the effective boundary dividing the cable cross section in two equal portions.

Parallel field measurements helped to reveal the fact that the twist pitches of the cable were quite different from what it originally were thought to be, which was proven by direct measurement of the twist pitches on the cable. Also the theory [2] was confirmed, that the signal . from the sensor located in the center of the conductor has a different sign from the sensors located in the valley. 


\section{SUMMARY}

Internal co-wound voltage sensors have been proposed and tested as part of the quench detection R\&D program for TPX. The NRE has verified the ability of these sensors to reduce the inductive noise to an acceptable level from all magnetic sources that the TPX magnets will be exposed to. Before this experiment was performed; the initial sensor location of choice was III, the center of the last stage subcable. Where it has an adequate rejection capacity for transverse fields, the performance of location I (in the triplex) is far superior for the parallel and self-fields. With the demise of the TPX project, ITER should seriously consider further development and inclusion of these sensors for the ITER Coils.

TABLE II

NRE RESULTS SUMMARY

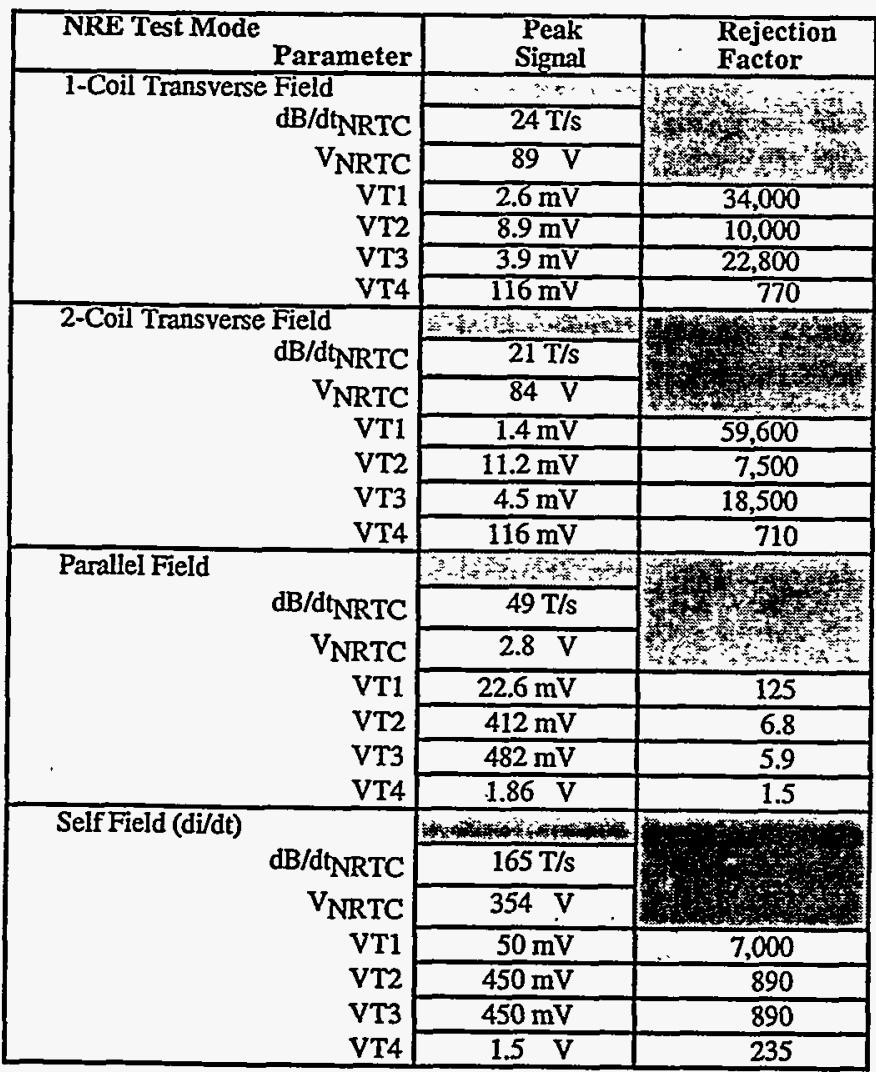

\section{ACKNOWLEDGMENT}

The authors would like to thank Jeff Moller, Mark Fowler, Andy Hinz, and Nate Liggins at LLNL for their contributions to the construction and setup of the experiment; JAERI for allowing LLNL to use their power supply to perform the NRE, Joel Schultz at MIT and Jon Zbasnik at LLNL for valuable discussions.

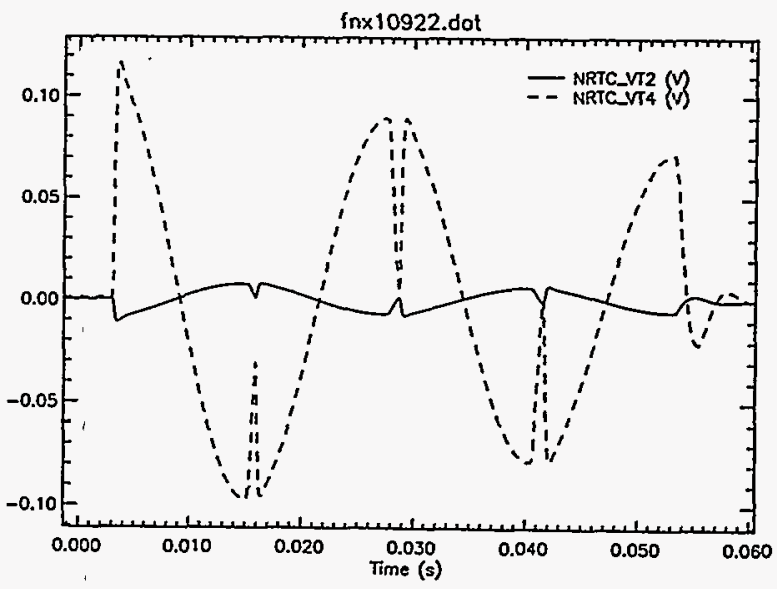

Fig 5. 2-Coil Transverse-Field NRE Data, showing signal from the sensor in the cable center (VT4) much higher than that from the sensor in the valley

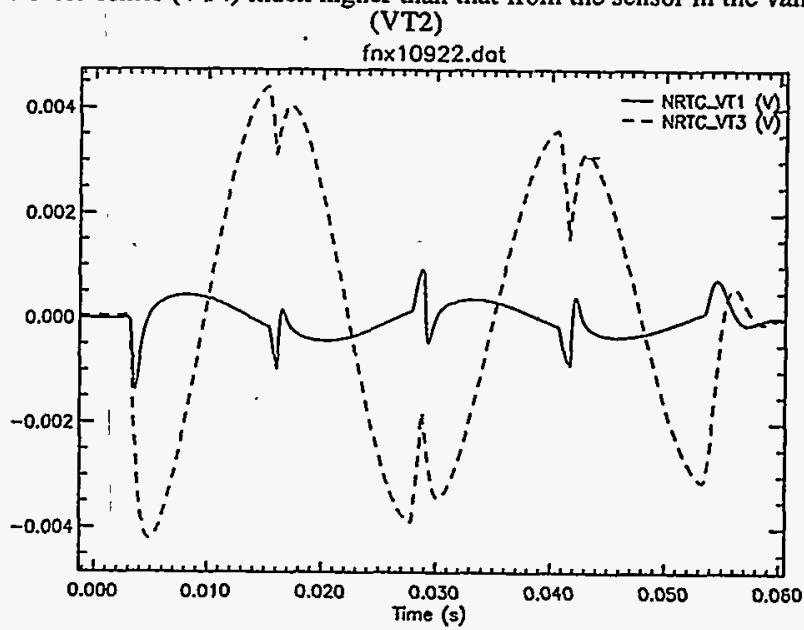

Fig. 6. 2-Coil Transverse-Field NRE Data, same shot as in Fig. 6, showing much better compensation of the inductive signal for sensors located in the first triplet (VTI) and in the center of the last stage subcable (VT3), both better than VT4 and VT2 in Fig.6. with VT1 much superior than the others.

\section{REFERENCES}

[1] G Deis, et. al., "TPX superconducting tokamak magnet system 1995 design and status overview," 16th IEEE/NPSS Symposium on Fusion Engineering, Champaign, IL,Sept, 30, 1995, in press.

[2] N.N. Martovetsky, M.R. Chaplin, "Normal-zone detection in tokamak superconducting magnets with co-wound voltage sensors", 14th Intl. Conf. on Magnet Tech., Tampere, Finland, June 11-16, 1995, in press.

[3] P.W. Wang, "12 V startup with a maximum field null at the breakdown for TPX," TPX Doc.\#14-940627-MTT-PWang-01, PPPL, June, 1994.

[4] A.L. Radovinsky, "Max. field \& time derivatives in the TPX TF coils during plasma startup \& plasma disruption, an update," TPX Doc.\# 13-940725-MIT-ARadovinsky-01, PPPL, July, 1994.

[5] J.H. Schultz, et. al."'Feasibility of the TPX voltage sensor quench detection system," TPX Memo \# 1314-950818-MIT-JSchultz-01, PPPL, August 18, 1995.

[6] I. Katz, et. al., "TPX structural \& cryogenic design criteria," TPX Doc. \# 94-950606-PPPL-IZatz-02, PPPL, June 6, 1995.

[7] M.R. Chaplin, "Noise-Rejection Experiment for TPX Ouench Detection R\&D, rev. 1," TPX Doc.\# 13-941110-LLNL-MChaplin-01, PPPL, November, 1994.

[8] M.R. Chaplin, "Results from the LLNL Noise-Rejection Experiment," TPX Memo \# 1314-950808-LLNL-MChaplin-01, PPPL, Aug-ust 18, 1995. 